\title{
PERFIL DAS NUTRIZES ADOLESCENTES E CARACTERÍSTICAS RELACIONADAS AO ALEITAMENTO MATERNO EM UMA CIDADE DO SUL DO BRASIL
}

\author{
Guilherme Tavares de Arruda \\ Áureo Júnior Weschenfelder ${ }^{1}$ \\ Melissa Medeiros Braz \\ Hedioneia Maria Foletto Pivetta ${ }^{2}$
}

\begin{abstract}
ARRUDA, G. T. de; WESCHENFELDER, A. J.; BRAZ, M. M.; PIVETTA, H. M. F. Perfil das nutrizes adolescentes e características relacionadas ao aleitamento materno em uma cidade do sul do Brasil. Arq. Cienc. Saúde UNIPAR, Umuarama, v. 22, n. 1, p. 23-26, jan./ abr. 2018.
\end{abstract}

\begin{abstract}
RESUMO: Este estudo objetivou analisar o perfil de nutrizes adolescentes e as características relacionadas ao aleitamento materno desta população em uma cidade do sul do Brasil. Trata-se de uma pesquisa quantitativa transversal, com abordagem exploratória e descritiva, realizada com 108 adolescentes nutrizes de uma cidade do sul do Brasil. As adolescentes foram entrevistas por meio de um questionário padronizado e validado que continha questões sobre aspectos socioeconômicos e de amamentação. A análise dos dados envolveu procedimentos de estatística descritiva. A maioria das mulheres possuía baixos níveis de escolaridade ( $\mathrm{n}=72 ; 66,67 \%)$ e nível socioeconômico ( $\mathrm{n}=70 ; 64,81 \%)$, ocupava-se do lar $(\mathrm{n}=59 ; 54,63 \%)$, era solteira ou vivia com o companheiro $(\mathrm{n}=42 ; 38,89 \%)$ e, apesar de ter recebido orientações sobre o aleitamento materno $(\mathrm{n}=93 ; 86,11 \%)$, o tempo de aleitamento materno exclusivo ( $\mathrm{n}=101 ; 44,44 \%)$ foi aquém do preconizado. Fissura/dor mamilar $(\mathrm{n}=35 ; 21,47 \%)$ e pouco leite $(\mathrm{n}=26 ; 15,95 \%)$ foram os fatores mais citados que dificultaram ou impediram o aleitamento materno. Esta pesquisa permitiu conhecer o perfil socioeconômico e as características relacionadas ao aleitamento materno na população estudada. Observou-se necessidade de maior atenção aos aspectos que envolvam o aleitamento materno por parte dos profissionais da saúde, fornecendo orientações e esclarecendo dúvidas sobre as práticas e cuidados para a amamentação.
\end{abstract}

PALAVRAS-CHAVE: Adolescente. Aleitamento materno. Gravidez na adolescência.

\section{PROFILE OF LACTATING ADOLESCENTS AND BREASTFEEDING CHARACTERISTICS IN A CITY OF SOUTHERN} BRAZIL

\begin{abstract}
This study aimed to analyze the profile of adolescent mothers and the breastfeeding characteristics related to this population in a city in the south of Brazil. This is a cross-sectional quantitative research, with an exploratory and descriptive approach, carried out on 108 adolescents from a city in the south of Brazil. The adolescents were interviewed through a standardized and validated questionnaire containing questions regarding socioeconomic and breastfeeding aspects. Data analysis involved descriptive statistics procedures. Most of the women had low schooling $(\mathrm{n}=72,66.67 \%)$ and low socioeconomic level $(\mathrm{n}=70,64.81 \%)$, took care of home $(\mathrm{n}=59 ; 54.63 \%)$, were single or lived with their partner $(n=42 ; 38.89 \%)$ and despite having received guidance on breastfeeding $(n=93 ; 86.11 \%)$, the time of exclusive breastfeeding $(\mathrm{n}=101 ; 44.44 \%)$ was lower than recommended. Nipple fissures or pain $(\mathrm{n}=35 ; 21.47 \%)$ and not enough milk ( $\mathrm{n}=26$; $15.95 \%$ ) were the most common factors hindering or preventing breastfeeding. This study enabled the authors to know the socioeconomic profile and breastfeeding characteristics of the studied population. Further care must be taken in all aspects involving breastfeeding, guided by health professionals providing clarifications and guidance regarding breastfeeding practices and care.
\end{abstract}

KEYWORDS: Adolescent. Breastfeeding. Pregnancy in adolescence.

\section{Introdução}

A adolescência é o período de vida compreendido entre os 10 e 19 anos de idade (WHO, 1995), e está relacionada à presença de transtornos psicossociais, doenças sexualmente transmissíveis e problemas relacionados à gravidez, ao parto e ao puerpério (QUIROGA; VITALLE, 2013). Conforme dados do Departamento de Informática do SUS (DATASUS), no Brasil, quase $20 \%$ dos partos realizados em 2014 foram de mães adolescentes. No Estado do Rio Grande do Sul, a taxa foi de 15,66\% (BRASIL, 2014). Esse é um dado que merece atenção, visto que a gestação nessa faixa etária é considerada de alto risco e pode estar relacionada a dificuldades no aleitamento materno (AM) (MARANHÃO et al., 2015).

Sabe-se que o AM traz inúmeros benefícios ao binômio mãe-bebê. Para o bebê, principalmente, o leite materno protege contra doenças, reduz a chance de obesidade e promove o desenvolvimento e crescimento infantil. Entre as vantagens para a mãe, o AM protege contra o câncer de mama, pode evitar nova gravidez nos seis primeiros meses após o parto e promove maior vínculo mãe-filho (BRASIL, 2009a). Apesar dessas vantagens, os fatores que dificultam ou impedem o AM ainda são realidades atuais, principalmente entre mães adolescentes (MARANHÃO et al., 2015).

$\mathrm{Na}$ literatura, são mencionados diversos fatores que relacionam a dificuldade no aleitamento à idade materna, dentre eles o nível de instrução das adolescentes e sua condição socioeconômica (SOUZA et al., 2012). Além desses, a falta de apoio emocional, a imaturidade, a ocupação com estudos e a anatomia das mamas também são bastante relatados e podem comprometer a saúde da mãe e do bebê (MARANHÃO et al., 2015).

Considera-se que os fatores que dificultam ou impedem a amamentação, relacionados à baixa faixa etária da mãe, sejam motivos de impacto para a saúde do bebê, tor-

DOI: 10.25110 /arqsaude.v22i1.2018.6255

${ }^{1}$ Acadêmicos do Curso de Fisioterapia, Universidade Federal de Santa Maria, Curso de Fisioterapia (UFSM), Santa Maria, RS.

${ }^{2}$ Professoras Adjuntas do Departamento de Fisioterapia e Reabilitação, Universidade Federal de Santa Maria (UFSM), Santa Maria, RS.

Autor correspondente: Guilherme Tavares de Arruda. Endereço: Rua General Osório, 330, Santa Maria, RS. CEP: 97060270. Telefone: (55) 99136-0953.

E-mail: gui_tavares007@hotmail.com 
nando-se um problema de saúde pública. Diante disso, o objetivo desse estudo consiste em analisar o perfil de nutrizes adolescentes e as características relacionadas ao AM desta população em uma cidade do sul do Brasil.

\section{Métodos}

Trata-se de uma pesquisa quantitativa transversal, com abordagem exploratória e descritiva, na qual foram utilizados dados do projeto de pesquisa intitulado "Influência dos fatores biológicos e ambientais no aleitamento materno exclusivo", desenvolvido junto a duas Unidades Básicas de Saúde e vinte e duas Unidades de Estratégia de Saúde da Família da cidade de Santa Maria/RS. Esta pesquisa foi aprovada no Comitê de Ética em Pesquisas da Universidade Federal de Santa Maria sob parecer 129.893.

A coleta de dados ocorreu nos dias de Campanha de Vacinação Contra a Poliomielite. Foram incluídas mulheres, mães de crianças de 0 a 2 anos de idade, residentes em Santa Maria/RS, que acessaram as Unidades de Saúde e postos volantes do município nos dias de Campanha. Foram excluídas as que não aceitaram responder o instrumento de pesquisa, e crianças acompanhadas por outro familiar senão a mãe no momento da coleta.

$\mathrm{O}$ instrumento de pesquisa era composto por questões relacionadas à mãe e à amamentação. As questões relativas à mãe envolviam características socioeconômicas. As perguntas sobre a amamentação continham informações como: orientações quanto ao AM; se a mãe amamentou o bebê; tempo de aleitamento materno exclusivo (AME); e fatores que dificultaram ou impediram o AM.

Para o cálculo do tamanho da amostra do projeto original para o desfecho AME, foram utilizados os seguintes parâmetros: intervalo de confiança (IC) $95 \%$, poder de $80 \%$, prevalência de AME 34\%, conforme pesquisa realizada pelo município de Santa Maria/RS por ocasião II Pesquisa de prevalência de aleitamento materno nas Capitais Brasileiras e Distrito Federal (BRASIL, 2009b) e RR1:2, obtendo-se uma amostra de 1.015. Devido ao insuficiente preenchimento de alguns formulários a amostra final foi de 905 questionários. Com isso, foi feito um recorte com as mães adolescentes, totalizando 108 mulheres.

Após a vacinação da criança, foi realizada uma entrevista com as mães por meio de um questionário padronizado e pré-codificado. A entrevista somente foi efetivada após o aceite e assinatura do Termo de Consentimento Livre e Esclarecido (TCLE), em atendimento à Resolução 466/2012 do Conselho Nacional de Saúde. Um teste piloto com dez mães foi realizado anteriormente à coleta, a fim de validar o questionário.

Após revisão e codificação dos dados, os mesmos foram duplamente digitados e, posteriormente comparados, no Excel 2013, para evitar perdas e/ou duplicidade dos dados. A análise dos dados envolveu procedimentos de estatística descritiva.

\section{Resultados}

Participaram do estudo 108 adolescentes nutrizes com média de idade de 17,51 $\pm 1,31$ anos. Houve predomínio de adolescentes brancas $(59,26 \%)$, com ensino fundamental
$(66,67 \%)$, solteiras $(38,89 \%)$ ou que moram com companheiro $(38,89 \%)$, do lar $(54,63 \%)$ e pertencentes à classe social $\mathrm{C}$ $(64,81 \%)$ (Tabela 1$)$.

Sobre as características relacionadas à amamentação, foram prevalentes as nutrizes que receberam orientações sobre AM $(86,11 \%)$ e amamentaram seu bebê por menos de seis meses de idade $(44,44 \%)$, característica do desmame precoce. Quanto aos fatores que dificultaram ou impediram o $\mathrm{AM}, 67,59 \%$ das adolescentes relataram um ou mais fatores, dentre eles: fissuras e dor nos mamilos $(21,47 \%)$ e pouco leite $(15,95 \%)$ foram os mais relatados (Tabela 2 ).

Tabela 1: Caracterização socioeconômica das adolescentes nutrizes de Santa Maria, Rio Grande do Sul (n=108).

\begin{tabular}{lcc}
\hline \multicolumn{1}{c}{ Características } & n & \% \\
\hline Cor autodeclarada & & \\
$\quad$ Branca & 64 & 59,3 \\
Preta & 17 & 15,7 \\
Parda ou amarela & 27 & 25 \\
\hline Escolaridade & & \\
$\quad$ Ensino fundamental & 72 & 66,7 \\
\hline Ensino médio & 32 & 29,7 \\
Ensino superior incompleto & 02 & 1,8 \\
\hline Ensino superior completo & 02 & 1,8 \\
Sem escolaridade & 0 & 0 \\
\hline Situação conjugal & & \\
\hline Solteira & 42 & 38,9 \\
\hline Casada & 21 & 19,4 \\
\hline Mora com companheiro & 42 & 38,9 \\
\hline Separada & 03 & 2,8 \\
\hline Ocupação & & \\
\hline Dor lar & 59 & 54,6 \\
Estudante & 28 & 25,9 \\
\hline Trabalhando & 18 & 16,7 \\
\hline Desempregada & 03 & 2,8 \\
\hline
\end{tabular}

\section{Classe social}

\begin{tabular}{lcc}
$\mathrm{A} 1-\mathrm{A} 2$ & 0 & 0 \\
$\mathrm{~B} 1-\mathrm{B} 2$ & 25 & 23,1 \\
$\mathrm{C} 1-\mathrm{C} 2$ & 70 & 64,8 \\
$\mathrm{D}$ & 10 & 9,3 \\
$\mathrm{E}$ & 03 & 2,8 \\
\hline
\end{tabular}

Tabela 2: Características relacionadas à amamentação entre as adolescentes nutrizes $(n=108)$ e fatores que dificultaram ou impediram o AM ( $\mathrm{n}=73)$.

\begin{tabular}{lcc}
\hline \multicolumn{1}{c}{ Características } & $\mathbf{n}$ & $\mathbf{\%}$ \\
\hline Recebeu orientações sobre AM & & \\
Sim & 93 & 86,1 \\
Não & 15 & 13,9 \\
\hline Você amamentou este bebê & & \\
Sim & 101 & 93,5 \\
Não & 07 & 6,5 \\
\hline
\end{tabular}




\begin{tabular}{|lcc|}
\hline Tempo de AME nesta gestação & & \\
\hline Não lembra & 06 & 5,5 \\
\hline Não houve AME & 34 & 31,5 \\
\hline Menos de seis meses & 48 & 44,5 \\
\hline Seis meses & 14 & 13 \\
\hline Mais de seis meses & 06 & 5,5 \\
\hline
\end{tabular}

Fatores que dificultaram ou impediram o AM nesta gestação*

\begin{tabular}{|lcc|}
\hline Fissuras e dor no mamilo & 35 & 21,5 \\
\hline Pouco leite & 26 & 16 \\
\hline Ingurgitamento mamário & 17 & 10,4 \\
\hline Leite fraco & 15 & 9,2 \\
\hline Demora da saída do leite & 13 & 8 \\
\hline Mamilo plano ou invertido & 13 & 8 \\
\hline Choro & 12 & 7,4 \\
\hline Reflexo de ejeção exacerbado & 10 & 6,1 \\
\hline Trabalho e licença à maternidade & 06 & 3,7 \\
\hline Sucção fraca & 05 & 3,1 \\
\hline Mastite & 04 & 2,4 \\
\hline $\begin{array}{l}\text { Tristeza após o nascimento da } \\
\text { criança }\end{array}$ & 04 & 2,4 \\
\hline Contraindicações ao AM & 02 & 1,2 \\
\hline Preocupação com o corpo & 01 & 0,6 \\
\hline AM: aleitamento materno; AME: aleitamento materno exclusivo. \\
*Mais de uma alternativa poderia ser assinalada.
\end{tabular}

\section{Discussão}

Neste estudo observou-se que, dentre os fatores que dificultaram ou impediram o AM, houve maior prevalência de dor e fissura no mamilo, seguido queixa de pouco leite. Dados semelhantes foram encontrados em estudo (MARQUES et al., 2008), no qual 151 mães adolescentes foram questionadas sobre as dificuldades na amamentação. Os autores verificaram que $41,7 \%$ das entrevistadas relataram alguma dificuldade. Dentre elas, as mais citadas foram dificuldade na pega do mamilo pelo bebê, fissura mamilar e leite insuficiente.

Sabe-se que a pega incorreta e a higiene inadequada das mamas podem causar fissuras mamilares, levando à dor e, assim, interrompendo a amamentação (NEVES et al., 2016). Além disso, leite insuficiente também pode estar associado à pega incorreta. Ao mamar insuficientemente, o bebê não satisfaz sua fome, querendo mamar por várias vezes seguidas. Com o tempo, ele pode acabar recusando o peito, dando a impressão à mãe de seu leite ser fraco ou insuficiente (MARQUES et al., 2008).

Diante disso, orientar as mães quanto ao cuidado das mamas e à estimulação da pega correta pode ser um importante fator para minimizar a interrupção na amamentação. Além dessas orientações pelos profissionais da saúde, é válido destacar o cuidado da saúde mental e emocional destas mães, para que a prática da amamentação seja algo prazeroso e promova vínculo entre mãe e bebê.

No presente estudo, apesar do tempo de AME ter sido de 6 meses em apenas 12,96\% das adolescentes, 86,11\% delas receberam orientações sobre o AM. O tempo de AME possui associação com orientações recebidas sobre o AM. Ou seja, mães que recebem orientações sobre AM no pré-natal aprendem a lidar com possíveis intercorrências durante o AM, o que reduziria as chances de desmame precoce. Tais dados contradizem a literatura (BARBIERI et al., 2015; SPINDOLA et al., 2014). É possível que a ocorrência de vínculo entre as nutrizes e os profissionais da saúde durante a consulta de pré-natal seja essencial para gerar confiança na mãe em aderir às recomendações transmitidas sobre o AM (SANTOS et al., 2016; SPINDOLA et al., 2014).

Nesta pesquisa, a maioria das nutrizes adolescentes declarou-se branca, com ensino fundamental, solteira ou morando com companheiro, do lar e pertencente à classe social C. Esses dados são similares aos encontrados em outro estudo (GUSMÃO et al., 2013), no qual a maioria das adolescentes tinha de 4 a 7 anos de escolaridade (ensino fundamental), era casada ou vivia com companheiro e pertencia à classe social C.

Em relação à situação conjugal das adolescentes, mães que moram com o companheiro amamentam por mais tempo, o que revela que a presença do pai pode influenciar no AM (COSTA et al., 2017). No presente estudo, por termos optado em utilizar somente estatística descritiva para análise dos dados, não se pode fazer maiores inferências sobre o tempo de AM e o estado civil desta amostra. Entretanto, verificou-se que o tempo de AME foi aquém do preconizado (WHO, 2015) entre a maioria das mulheres.

O baixo nível educacional e a condição socioeconômica inferior são fortes fatores relacionados à gravidez na adolescência. Nesta população, a gestação pode causar interrupção dos estudos, tornando a adolescente pouco qualificada ao trabalho e com nível de conhecimento baixo, levando-a, assim, ao cuidado do lar (SANTOS et al., 2016). Evidentemente, o incentivo ao uso de métodos contraceptivos poderia reduzir os índices de gravidez na adolescência e influenciar positivamente no nível educacional dessa população.

Entre as limitações do estudo, destaca-se que foi utilizada somente a estatística descritiva para a análise dos dados. Além disso, por se tratar de estudo transversal, as características relacionadas ao AM se remeteram com maior precisão ao momento da entrevista, visto que o viés de memória pode comprometer a exatidão das informações. A entrevista, técnica utilizada para a coleta de dados, também apresenta limitações, como a inadequada compreensão das perguntas e o fornecimento de respostas falsas.

\section{Conclusão}

Este estudo permitiu conhecer o perfil socioeconômico e as características relacionadas ao AM entre nutrizes adolescentes de uma cidade do sul do Brasil. Verificou-se que a maioria das mulheres possuía baixos níveis de escolaridade e socioeconômico, ocupava-se do lar, era solteira ou vivia com o companheiro e, apesar de ter recebido orientações sobre o AM, o tempo de AME foi aquém do preconizado. Além disso, fissura, dor mamilar e pouco leite foram os fatores mais citados que dificultaram ou impediram o AM e parecem ter contribuído para o baixo tempo de AME. 
Nesse sentido, torna-se necessário conhecer o perfil desta população e dar maior atenção aos aspectos que envolvam o AM, fornecendo orientações e esclarecendo dúvidas sobre as práticas e cuidados para a amamentação, de forma a promover o AM.

Sugere-se a realização de estudos longitudinais para seguimento das gestantes, a fim de delinear, com maior fidedignidade, a tendência comportamental do AM em adolescentes do município. Para estudos futuros, recomenda-se a utilização de diferentes instrumentos de pesquisa, como registros do sistema de saúde, prontuários, questionários com maior confiabilidade ou instrumentos baseados na observação da mamada, que possibilitem a identificação das características relacionadas ao AM entre adolescentes, qualificando a assistência materno-infantil para esta população.

\section{Referências}

BARBIERI, M. C. et al. Aleitamento materno: orientações recebidas no pré-natal, parto e puerpério. Semin: Cien. Biol. Saúde, v. 36, n. 1, p. 17-24, 2015.

BRASIL. Ministério da Saúde. Secretaria de Atenção à Saúde. II pesquisa de prevalência de aleitamento materno nas capitais brasileiras e Distrito Federal. Brasília: Ministério da Saúde, 2009a.

- Secretaria de Atenção à Saúde.

Departamento de Atenção Básica. Saúde da criança: nutrição infantil: aleitamento materno e alimentação complementar. Brasília, 2009b.

. Secretaria Executiva Departamento de Informática do SUS/DATASUS. Informações de Saúde. Estatísticas vitais: Nascidos Vivos, 2014.

COSTA, R. S. L. et al. Dificuldades encontradas pelas mães ao amamentar em uma Unidade de Referência em Atenção Primária. DêCiência em Foco, v. 1, n. 1, p. 48-63, 2017.

GUSMÃO, A. M. et al. Prevalência de aleitamento materno exclusivo e fatores associados: estudo transversal com mães adolescentes de 14 a 16 anos em Porto Alegre, RS, Brasil.

Ciênc. Saúde Coletiva, v. 18, n. 11, p. 3357-68, 2013.

MARANHÃO, R. A. et al. Fatores associados ao aleitamento materno exclusivo entre mães adolescentes. Cad. Saúde Colet. v. 23, n. 2, p. 132-39, 2015.

MARQUES, R. F. S. V. et al. Fatores relacionados às dificuldades no aleitamento materno entre mães adolescentes da Fundação Santa Casa de Misericórdia do Pará. Rev. Para. Med. v. 22, n. 1, p. 57-62, 2008.

NEVES, B. R. et al. Intercorrências mamárias relacionadas com à amamentação: uma revisão sistemática. Rev. Ciên.

Saúde Oeste Baiano - Higia, v. 1, n. 2, p. 58-73, 2016.

ORGANIZÁCION MUNDIAL DE LA SALUD (OMS). La salud de los jóvenes: um reto y uma esperanza. Genebra: OMS, 1995.
QUIROGA, F. L.; VITALLE, M. S. S. O adolescente e suas representações sociais: apontamentos sobre a importância do contexto histórico. Physis, v. 23, n. 3, p. 863-878, 2013.

SANTOS, L. P. S. et al. Conhecimento, atitude e prática de puérperas adolescentes em relação ao aleitamento materno. Adolesc. Saude, v. 13, n. 1, p. 7-18, 2016.

SOUZA, S. N. D. H. et al. Prevalência de aleitamento materno e fatores associados no município de Londrina-PR. Acta Paul Enferm. v. 25, n. 1, p. 29-35, 2012.

SPINDOLA, T. et al. Breastfeeding during adolescence: life history of first-time mothers. J. Res.: Fundam. Care. v. 6, n. 1, p. 414-424, 2014.

WORLD HEALTH ORGANIZATION (2015). WHO global data bank on infant and young child feeding. Disponível em: <http://www.who.int/nutrition/databases/infantfeeding/ en>. Acesso em: 17 abr. 2017. 